\title{
Critical Mutation Rate Has an Exponential Dependence on Population Size
}

\author{
Alastair Channon ${ }^{1}$, Elizabeth Aston ${ }^{1}$, Charles Day ${ }^{1}$, Roman V. Belavkin ${ }^{2}$ and Christopher G. Knight ${ }^{3}$ \\ ${ }^{1}$ Research Institute for the Environment, Physical Sciences and Applied Mathematics, Keele University, ST5 5BG, UK \\ ${ }^{2}$ School of Engineering and Information Sciences, Middlesex University, London, NW4 4BT, UK \\ ${ }^{3}$ Faculty of Life Sciences, The University of Manchester, M13 9PT, UK \\ \{a.d.channon,e.j.aston,c.r.day\}@epsam.keele.ac.uk, r.belavkin@mdx.ac.uk, chris.knight@manchester.ac.uk
}

\begin{abstract}
Populations of individuals exist in a wide range of sizes, from billions of microorganisms to fewer than ten individuals in some critically endangered species. In any evolutionary system, there is significant evolutionary pressure to evolve sequences that are both fit and robust; at high mutation rates, individuals with greater mutational robustness can outcompete those with higher fitness, a concept that has been referred to as survival-of-the-flattest. Previous studies have not found a relationship between population size and the mutation rate that can be tolerated before fitter individuals are outcompeted by those that have a greater mutational robustness. However, using a genetic algorithm with a simple two-peak fitness landscape, we show that the mutation rates at which the high, narrow peak and the lower, broader peak are lost for increasing population sizes can be approximated by exponential functions. In addition, there is evidence for a continuum of mutation rates representing a transition from survival-of-the-fittest to survival-of-the-flattest and subsequently to the error catastrophe. The effect of population size on the critical mutation rate is shown to be particularly noticeable in small populations. This provides new insight into the factors that can affect survival-of-the-flattest in small populations, and has implications for populations under threat of local extinction.
\end{abstract}

\section{Introduction}

Biological population sizes can range from small numbers of individuals to very large numbers of individuals. For example, RNA viruses can reach population sizes of around $10^{10}$ in a short amount of time (Comas et al., 2005), whereas some animal species may exist in populations consisting of only hundreds or even fewer than ten individuals in some critically endangered species saved on the brink of extinction. A population of genomes constantly evolves through the processes of mutation, recombination (in sexual reproduction), selection and genetic drift (Hartl and Clark, 2007). Population dynamics can be modelled in silico using genetic algorithms, in which populations of sequences are allowed to undergo mutation, recombination and selection at specified rates; studies can be done in a controlled environment within time-frames not possible in many natural biological systems, producing results that are comparable both to theory and to experimental results in microorganisms.
In any evolutionary system, including genetic algorithms and natural biological systems, there is significant evolutionary pressure to evolve sequences that are both fit and robust (Jones and Soule, 2006). Robustness is defined as the average effect of a specified perturbation (such as a new mutation) on the fitness of a specified genotype (Masel and Trotter, 2010). The more robust a genotype, the smaller the effect of mutation on fitness; in systems with high levels of mutation, robustness can reduce the negative effects of deleterious mutation. Smaller populations are more susceptible to loss of fitness through random genetic drift (Comas et al., 2005; Hartl and Clark, 2007). Therefore it is expected that population size should influence the size of mutation rate that can be tolerated before fitter individuals are outcompeted by those with a greater mutational robustness.

\section{Mutational Robustness and Survival-of-the-flattest}

The concept of a fitness landscape was introduced by Wright (1932) and later combined with the notion of sequence space by Eigen and Schuster (1979). Each sequence in sequence space has a fitness value, which represents its relative replication capacity (Domingo and Wain-Hobson, 2009). Fitness landscapes are sometimes considered to resemble mountain ranges, with the fittest sequences at the peaks. However, the concept requires a more careful interpretation in high dimensional sequence spaces with low alphabet size, such as nucleic acids, which have an alphabet size of four (in that they are sequences consisting of four possible units, A, C, G and $\mathrm{T}$ ). For example, the space of $\mathrm{N}$-length binary sequences is an $\mathrm{N}$-dimensional hypercube rather than a 3-dimensional Euclidean geometry, and can only be represented as such by use of a reductive transform between the two spaces. Exploration of sequence space is done through evolution by mutation, recombination and selection in accordance to the fitness landscape. Selection increases the frequencies of the fittest sequences, while mutation introduces variation, often at a cost to individual fitness. The balance between these two forces is referred to as the mutation-selection balance (Kimura and Maruyama, 1966; Bull et al., 2005). A popula- 
tion in mutation-selection balance will tend to cluster around the fitness peaks and form what is known as a quasispecies (Eigen and Schuster, 1979; Bull et al., 2005; Nowak, 2006).

In a landscape with a single fitness peak, a quasispecies is able to maintain its position surrounding the top of the peak so long as the mutation rate does not exceed a particular rate known as the error threshold. Above this threshold, there is an error catastrophe and the population delocalizes across sequence space (Tannenbaum and Shakhnovich, 2004; Bull, 2005; Nowak, 2006; Takeuchi and Hogeweg, 2007; Domingo and Wain-Hobson, 2009; Schuster, 2009; Tejero et al., 2011).

The concept of error threshold was introduced in Eigen et al. (1988) and later in Nowak and Schuster (1989) based on the quasispecies equation:

$$
\dot{x}_{i}=\sum_{j=1}^{m} x_{j} f_{j} q_{j i}-\phi x_{i}
$$

Here, $x_{i}$ is the frequency of genotype number $i$, where $i \in\left[1, \ldots, \alpha^{n}\right], \alpha$ is the alphabet size, $n$ is the length of sequences, $\sum x_{i}=1, f_{j}$ is fitness (selection), $\phi=\sum x_{i} f_{i}$ is the average fitness, and $q_{j i}$ is a transition probability (mutation). The derivative in time is denoted $\dot{x}$, and there are $m$ genetic sequences.

Selection and mutation provide two forces (or pressures) on the population, and they can be combined into one matrix $\left(w_{j i}=f_{j} q_{j i}\right)$ (see Nowak (2006), p. 35). Selection draws the population closer to the highest fitness, while mutation is usually assumed to have deleterious effect due to which the population drifts away from the highest fitness. Generally, the population converges to a stable (equilibrium) state that is defined by an eigenvector of the mutation-selection matrix $\left(w_{j i}\right)$. This eigenvector corresponds to the largest eigenvalue of $\left(w_{j i}\right)$, which is the average fitness $\phi$.

The idea of an error threshold is based on the existence of a mutation-selection balance when the effect of mutation does not exceed that of the selection pressure. The corresponding value of the mutation rate is referred to as the error threshold, and it is the maximal mutation rate that allows a population to stay centred 'around' the fitness peak.

However, in landscapes with more than one peak, there may also be one (or more) critical mutation rates at which the population loses its ability to localize to fitter peaks, while potentially retaining its ability to remain on lower, flatter peaks (Wilke et al., 2001; Tannenbaum and Shakhnovich, 2004; Comas et al., 2005; Wilke, 2005). This represents a phase transition from survival-of-the-fittest to survival of those individuals with greater mutational robustness, a concept referred to as survival-of-the-flattest (Wilke et al., 2001; Bull et al., 2005; Comas et al., 2005; Wilke, 2005; Sanjuán et al., 2007; Sardanyés et al., 2008; Tejero et al., 2011). This concept is based on the idea that at low mutation rates, selection favours individuals in a quasispecies that reside at peaks with higher fitness, even if the peaks are steep and narrow, due to the rarity of mutations that push individuals off the peaks (Lenski et al., 2006). In contrast, at high mutation rates, selection favours individuals that reside at peaks less likely to result in off-peak mutations: individuals located in flatter regions of the fitness landscape are less likely to suffer large reductions in fitness compared with those that may be initially fitter but reside in parts of the landscape with steeper peaks. Individuals that are part of a neutral network (Kimura, 1983), in that they are surrounded by other individuals with equivalent fitness, are said to be mutationally robust (Bull et al., 2005; Bornberg-Bauer and Kramer, 2010; Wilke, 2001a; Wilke, 2001b); their fitness will be less sensitive to mutation than individuals that are not well connected.

Survival-of-the-flattest has been observed in digital organisms (Wilke et al., 2001; Sardanyés et al., 2008), theoretically (Wilke, 2001a; Sardanyés et al., 2008), in simulated RNA evolution (Wilke, 2001b), and in RNA viruses (Sanjuán et al., 2007). In addition, evolution of mutational robustness has been observed in simulated RNA evolution (van Nimwegan et al., 1999) and in laboratory protein evolution experiments (Bloom et al., 2007). Both van Nimwegan et al. (1999) and Bloom et al. (2007) place an emphasis on the degree of polymorphism in the population, suggesting that highly polymorphic populations are more likely to spread across many nodes of a neutral network (each corresponding to a genotype), concentrating at highly connected parts; individuals at highly connected nodes have greater robustness to mutation, which they pass on to the next generation. Robustness will evolve in any population where the product of the population size and frequency of mutation per sequence per generation is sufficiently large $(>1)$. Krakauer and Plotkin (2002) refer to flat landscapes as redundant, and steeper landscapes as antiredundant. They suggest that both in theory and in individual-based stochastic simulations, redundancy increases the mean fitness in small populations as it masks mutations that arise due to mutational drift. However, large populations are less affected by drift, and so are more able to occupy high-fitness peaks in sharp landscapes.

Wilke (2001b) ran simulations with population sizes as low as 100 and noted "that for very small populations, the predictive value of the differential equation approach diminishes". Later Wilke noted that his results agreed with Comas et al. (2005) in finding "that population size played only a minor role in determining the position of the critical mutation rate" (Wilke, 2005), within the context of their experiments. Comas et al. used population sizes as low as 250 and concluded "that the critical mutation rate was independent of population size" (Comas et al., 2005) despite the fact that there did appear to be some correlation for certain cases.

Jones and Soule (2006) determined that the role of genetic robustness in evolution differs significantly depending on whether it is a generational or steady state genetic algorithm that is being used. In a steady state algorithm, only 
a few individuals are replaced at a time, as opposed to a generational algorithm which replaces the entire population at once. Many studies that have confirmed the notion of survival-of-the-flattest have used generational models, such as Wilke et al.'s (2001) evolution of digital organisms in Avida, and Krakauer and Plotkin's (2002) study of redundancy and antiredundancy (Jones and Soule, 2006). Jones and Soule suggest that for evolutionary dynamics experiments, the class of algorithm used can have a significant effect on the observed outcome. They point to steady state algorithms as being of particular interest to the artificial life community, as natural evolution resembles the action of a steady-state-like algorithm: evolution in biological systems does not usually follow the generational approach of evolving every individual in the population synchronously.

However, the problem with steady state algorithms is that they typically allow individuals to survive on fitness peaks indefinitely. This is not a realistic property when modelling evolutionary dynamics. A preferable approach is to use a generational genetic algorithm which retains the key features of steady state evolution: fitness rank-based selection and a degree of asynchronicity. It should be noted that fitness in this sense refers to a score assigned to each individual based on a given fitness function, as opposed to the biological definition of fitness as a measure of replication rate; the exact fitness values used are unimportant as it is relative fitness that determines which individuals are selected. Rankbased selection (the assignment of reproductive fitness rates according to fitness score rank) overcomes the scaling problems of fitness score proportional selection (the assignment of reproductive fitness rates in proportion to fitness score), so creating a general model from a specific fitness score landscape such as that in figure 1, while retaining the key property that sequences with higher fitness scores have (probabilistically) more offspring than those with lower scores. This approach also allows for the existence of a critical mutation rate: with a standard steady state algorithm, always retaining the fittest individual prevents the population from ever losing the highest current peak.

\section{Simulation Model}

An individual sequence consists of a string of characters drawn from an alphabet of size 4 (which can be thought of as, for example, $\mathrm{A} / \mathrm{C} / \mathrm{G} / \mathrm{T}$ or $0 / 1 / 2 / 3$ ) with a fixed length of 30 . In each step of the algorithm, three individual sequences are selected at random from the population. Two of the three selected individuals are chosen as parents in a crossover which replaces the third individual with the resulting child. The child is then subject to one round of point mutation (to a different base) at a given per-base mutation rate. The individual to be replaced is decided each time based on the fitnesses of the three selected individuals: there is an equally small chance of either of the two fittest of the three being replaced $(25 \%)$, and a larger chance of replacing the

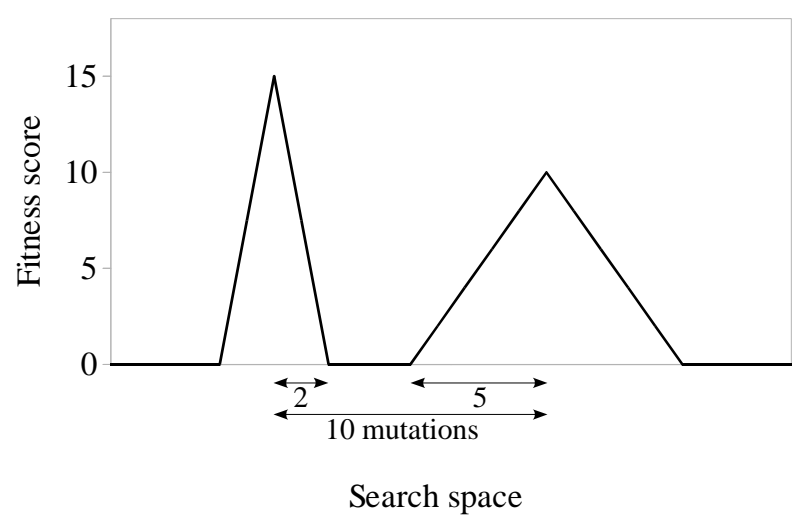

Figure 1: Two-peak fitness landscape, with one narrow peak of high fitness (peak 0), and one broader, flatter peak of lower fitness (peak 1).

least fit $(50 \%)$. This process continues until each individual in the population has been chosen exactly once; this represents one generation, and ensures that there is no chance of any individual avoiding being chosen and so remaining static in the landscape. The fitness of each individual sequence is evaluated based on a two-peak fitness landscape with one narrow peak of high fitness (peak 0), and a broader, flatter peak with lower fitness (peak 1) (figure 1). Peak 0 has a maximum fitness score of 15 and a radius (Hamming distance from top-of-peak to zero fitness score) of 2; peak 1 has a maximum fitness score of 10 and a radius of 5, with its top chosen as an arbitrary point (fixed throughout evolution) with a Hamming distance of 10 from the top of peak 0 . Individuals are allowed to move anywhere on the slopes, or in between the peaks. This is a simple landscape in which survival-of-the-flattest can occur. The effect of mutation on fitness is smaller within peak 1 than within peak 0 ; individuals located on peak 1 will have higher mutational robustness compared with those located on peak 0 .

Following the experimental procedure designed by Wilke et al. (2001) (and used by Comas et al., 2005) we initialized half of the population of sequences to be on top of the high, narrow peak, and the other half to be on top of the lower, flatter peak. This procedure prevents initialization bias between peaks. The simulation was run for 10,000 generations, and the number of generations it took to first lose each peak was recorded (where a peak was considered to be lost when there were no individuals present anywhere in its range). If a peak was never lost within the 10,000 generations, a value of 1 was recorded. A range of per-base mutation rates was tested for a range of population sizes. The simulation was run 2,000 times for each combination of mutation rate and population size. The mutation rate by which $95 \%$ of the runs had lost each peak was recorded, where a peak was considered to have not ever been lost only if there were individuals remaining on it at the end of the 10,000 generations. 


\begin{tabular}{|c|c|c|c|c|}
\hline Population size $(\mathrm{m})$ & Observed $\mu_{0}$ & Stretched Exponential $\epsilon_{0}$ & Difference $\delta_{0}\left(\mu_{0}-\epsilon_{0}\right)$ & Difference/Stretched Exp. $\left(\delta_{0} / \epsilon_{0}\right)$ \\
\hline \hline 10 & $0.150 \%$ & $0.150 \%$ & $0.000 \%$ & $0.1 \%$ \\
\hline 20 & $0.550 \%$ & $0.554 \%$ & $-0.004 \%$ & $-0.8 \%$ \\
\hline 30 & $0.750 \%$ & $0.742 \%$ & $0.008 \%$ & $1.0 \%$ \\
\hline 40 & $0.850 \%$ & $0.853 \%$ & $-0.003 \%$ & $-0.4 \%$ \\
\hline 50 & $0.925 \%$ & $0.926 \%$ & $-0.001 \%$ & $-0.1 \%$ \\
\hline 60 & $0.975 \%$ & $0.978 \%$ & $-0.003 \%$ & $-0.3 \%$ \\
\hline 70 & $1.025 \%$ & $1.017 \%$ & $0.008 \%$ & $0.8 \%$ \\
\hline 80 & $1.050 \%$ & $1.046 \%$ & $0.004 \%$ & $-0.4 \%$ \\
\hline 90 & $1.065 \%$ & $1.070 \%$ & $-0.005 \%$ & $-0.8 \%$ \\
\hline 100 & $1.080 \%$ & $1.089 \%$ & $-0.009 \%$ & $-0.2 \%$ \\
\hline 200 & $1.170 \%$ & $1.172 \%$ & $-0.002 \%$ & $0.3 \%$ \\
\hline 300 & $1.200 \%$ & $1.197 \%$ & $0.003 \%$ & $0.2 \%$ \\
\hline 400 & $1.210 \%$ & $1.207 \%$ & $0.003 \%$ & $0.2 \%$ \\
\hline 500 & $1.215 \%$ & $1.212 \%$ & $0.003 \%$ & $0.4 \%$ \\
\hline 600 & $1.220 \%$ & $1.215 \%$ & $0.005 \%$ & $0.7 \%$ \\
\hline 700 & $1.225 \%$ & $1.217 \%$ & $0.008 \%$ & $0.6 \%$ \\
\hline 800 & $1.225 \%$ & $1.218 \%$ & $0.007 \%$ & $-0.7 \%$ \\
\hline 900 & $1.210 \%$ & $1.219 \%$ & $-0.009 \%$ & $-1.2 \%$ \\
\hline 1000 & $1.205 \%$ & $1.219 \%$ & $-0.014 \%$ & \\
\hline
\end{tabular}

Table 1: Mutation rate $\mu_{0}$ by which $95 \%$ of runs lost peak 0 .

\section{Results}

The results (figure 2, and tables 1 and 2) show that population size affects the size of mutation rate required for the predominant outcome of the runs to shift from survivalof-the-fittest to survival-of-the-flattest, and that this is particularly noticeable in populations with 100 individuals or less. Similarly, the size of mutation rate required for approximately $95 \%$ of the runs to have lost both peaks also has a dependence on population size. The results of the simulation can be approximated by a simple exponential function: $y=A-B m^{C}$ for some values of the parameters $A, B$ and $C$, where $m$ is population size. However, they are more closely fitted by a stretched exponential function: $y=A-B * e^{-\left((m / C)^{D}\right)}$.

As opposed to there being instantaneous transitions from survival-of-the-fittest to survival-of-the-flattest and to the error catastrophe, at discrete mutation rates, there appear to be gradual transitions in which there are shifts in tendency from the first to the second, and from the second to the third. The mutation rate corresponding to $95 \%$ of the runs having lost the high, narrow peak (peak 0) within 10,000 generations marks a point at which the former transition (from survivalof-the-fittest to survival-of-the-flattest) is essentially complete. This can be considered as a critical mutation rate. For a population of 100 individuals, this is at a per-base mutation rate of approximately $1.08 \%$ (table 1). Figure 3(a) shows the number of generations taken to lose each peak at this mutation rate, for each of the 2,000 runs with population size 100 . Just $52 \%$ of these runs lost peak 1 within the dura-

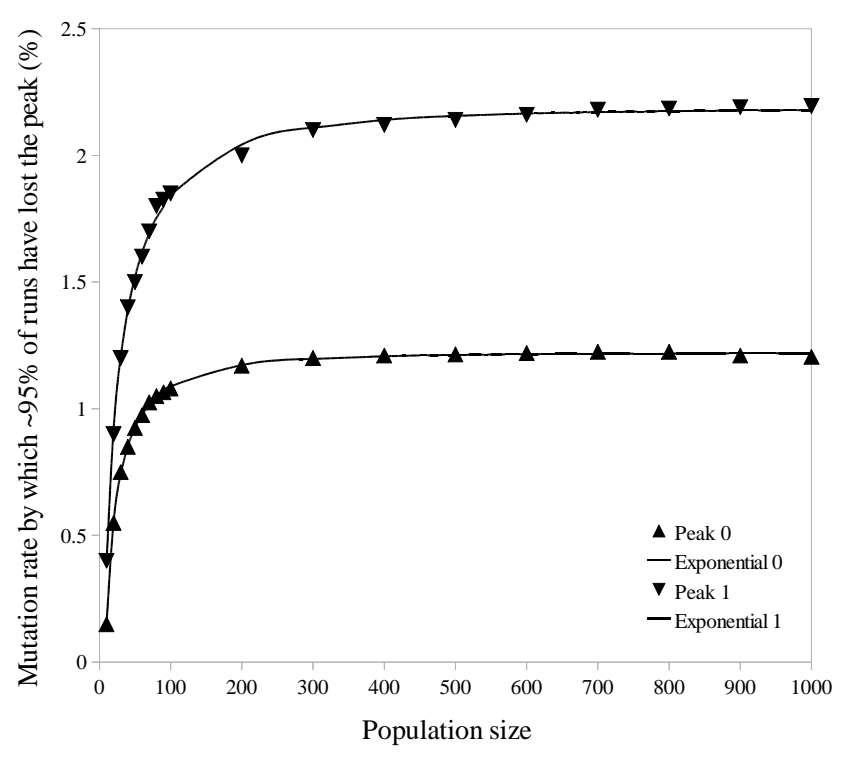

Figure 2: The results of the simulation for both peak 0 (high, narrow peak) and peak 1 (lower, flatter peak) can be approximated by an exponential function, where $y=$ $A-B * e^{-\left((m / C)^{D}\right)}$ (with $m$ being population size). The parameters obtained by curve-fitting using a least squares method were, for peak 0: $A=1.221 \%, B=7.001 \%, C$ $=1.440, D=0.3250$, and for peak 1: $A=2.184 \%, B=$ $5.438 \%, C=7.721, D=0.3978$. 


\begin{tabular}{|c|c|c|c|c|}
\hline Population size $(\mathrm{m})$ & Observed $\mu_{1}$ & Stretched Exponential $\epsilon_{1}$ & Difference $\delta_{1}\left(\mu_{1}-\epsilon_{1}\right)$ & Difference/Stretched Exp. $\left(\delta_{1} / \epsilon_{1}\right)$ \\
\hline \hline 10 & $0.400 \%$ & $0.389 \%$ & $0.011 \%$ & $2.8 \%$ \\
\hline 20 & $0.900 \%$ & $0.921 \%$ & $-0.021 \%$ & $-2.3 \%$ \\
\hline 30 & $1.200 \%$ & $1.206 \%$ & $-0.006 \%$ & $-0.5 \%$ \\
\hline 40 & $1.400 \%$ & $1.390 \%$ & $0.010 \%$ & $0.7 \%$ \\
\hline 50 & $1.500 \%$ & $1.520 \%$ & $-0.020 \%$ & $-1.3 \%$ \\
\hline 60 & $1.600 \%$ & $1.617 \%$ & $-0.017 \%$ & $-1.0 \%$ \\
\hline 70 & $1.700 \%$ & $1.692 \%$ & $0.008 \%$ & $0.4 \%$ \\
\hline 80 & $1.800 \%$ & $1.753 \%$ & $0.047 \%$ & $1.3 \%$ \\
\hline 90 & $1.825 \%$ & $1.802 \%$ & $0.023 \%$ & $0.4 \%$ \\
\hline 100 & $1.850 \%$ & $1.843 \%$ & $0.007 \%$ & $-2.1 \%$ \\
\hline 200 & $2.000 \%$ & $2.043 \%$ & $-0.043 \%$ & $-0.4 \%$ \\
\hline 300 & $2.100 \%$ & $2.109 \%$ & $-0.009 \%$ & $-0.9 \%$ \\
\hline 400 & $2.120 \%$ & $2.140 \%$ & $-0.020 \%$ & $-0.7 \%$ \\
\hline 500 & $2.140 \%$ & $2.156 \%$ & $-0.016 \%$ & $-0.2 \%$ \\
\hline 600 & $2.160 \%$ & $2.165 \%$ & $-0.005 \%$ & $0.4 \%$ \\
\hline 700 & $2.180 \%$ & $2.171 \%$ & $0.009 \%$ & $0.5 \%$ \\
\hline 800 & $2.185 \%$ & $2.174 \%$ & $0.011 \%$ & $0.6 \%$ \\
\hline 900 & $2.190 \%$ & $2.177 \%$ & $0.013 \%$ & $0.8 \%$ \\
\hline 1000 & $2.195 \%$ & $2.179 \%$ & $0.016 \%$ & \\
\hline
\end{tabular}

Table 2: Mutation rate $\mu_{1}$ by which $95 \%$ of runs lost peak 1 .

tion of the simulation (compared to $95 \%$ for peak 0 ). At this mutation rate, early loss of peak 0 appears to be a condition for survival-of-the-flattest. Loss of peak 0 is then followed by one of two events: either peak 1 is lost relatively quickly (within 200 generations) or it is maintained for the duration of the simulation. The fate of the population after loss of peak 0 is therefore dependent on whether or not it is able to quickly converge on peak 1. Figure 3(a) shows (at this mutation rate) that when peak 0 is not lost early, the number of generations taken to lose peak 0 is distributed approximately evenly up to 10,000 generations.

The mutation rate corresponding to $95 \%$ of the runs having lost the lower, flatter peak (peak 1) within 10,000 generations marks a point at which the latter transition (from survival-of-the-flattest to the error catastrophe) is essentially complete. This can be considered as another critical mutation rate (or the error threshold). For a population of 100 individuals, this is at a per-base mutation rate of approximately $1.85 \%$ (table 2). Figure 3(b) shows the number of generations taken to lose each peak at this mutation rate, for each of the 2,000 runs with population size 100. It is an apparent reversal of figure 3(a) but with $100 \%$ of the runs having lost peak 0 within 200 generations. The population has almost entirely lost the ability to localize to either peak.

\section{Discussion}

At high mutation rates, individuals with greater mutational robustness can outcompete those with higher fitness. Previous studies have not found a relationship between population size and the critical mutation rate, at which there is a phase transition from survival-of-the-fittest to survival-ofthe-flattest (Comas et al., 2005). However, the results of the current study suggest that population size does have an effect on the size of mutation rate that can be tolerated before the population loses the fittest and the flattest peaks, and that this is particularly noticeable in populations with 100 individuals or less. As shown in figure 2, the size of mutation rate at which each peak is lost for increasing population sizes can be approximated by an exponential function. One possible reason for this is that small populations are more susceptible to stochastic variation due to random genetic drift (Comas et al., 2005; Hartl and Clark, 2007); small populations with relatively large genomes cannot explore the entire neutral space of the landscape. Consequently, quasispecies formation is difficult, and the fitness peaks may be more easily lost. The dramatic reduction in critical mutation rate observed for small populations has implications for local extinction events in which there is a significant drop in population size. Further work will be necessary to apply this result to populations under threat of local extinction.

The dynamics of finite populations have very different properties compared to those of infinite populations, for example non-zero probability of extinction. The latter can be a good approximation of the former if the size of populations is large. However, where a small population size is fundamental to the issue of concern, as with the relationship established empirically in this paper, and in any work on extinction events (zero population size), such approximations break down. This situation is similar to statistical mechanics, where systems of large numbers of particles 


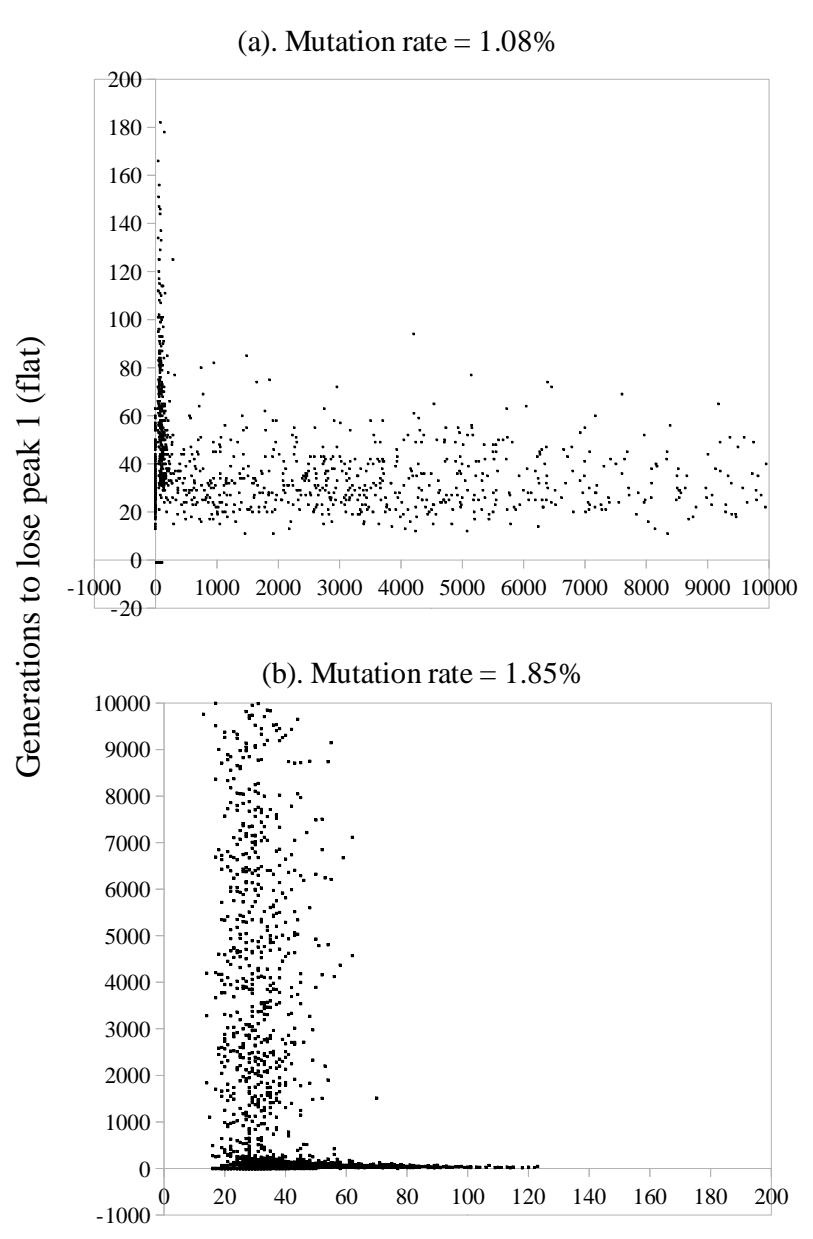

Generations to lose peak 0 (fit)

Figure 3: Transition from survival-of-the-fittest to survivalof-the-flattest and subsequently to the error catastrophe. Each point represents the number of generations it took to lose the high, narrow peak (peak 0) and the number to lose the lower, flatter peak (peak 1), in a single run of the GA for population size 100 . Where a peak was not lost within 10,000 generations, a value of -1 was assigned for that particular run of the GA: all points on the negative side of either axis should be taken to have a higher value than 10,000. (a) The mutation rate by which $95 \%$ of the runs had lost peak 0 within the duration of the simulation; just $52 \%$ of these runs lost peak 1 . This demonstrates that the transition from survival-of-the-fittest to survival-of-the-flattest is essentially complete. This can be considered as a critical mutation rate. (b) The mutation rate by which $95 \%$ of the runs had lost peak 1 within the duration of the simulation; $100 \%$ of these runs lost peak 0 . This demonstrates that the transition from survival-of-the-flattest to the error catastrophe is essentially complete, with the population having almost entirely lost the ability to localize to either peak. are approximated by laws derived for an infinite number of particles. The relation between the two is asymptotic and rooted in the law of large numbers. In fact, one can obtain equations for infinite populations from stochastic equations for finite populations by taking their expected value with respect to a probability measure on the population sizes $m \in\{0,1, \ldots\}$. The dynamics of finite populations can be described by stochastic differential equations. In particular, branching processes have been used to study the population dynamics of populations with variable (random) finite size (Jagers, 1975). The dynamics of finite populations have also been studied using the Moran process (Moran, 1962; Nowak, 2006). This work establishes an important empirical relationship between population size and critical mutation rate; the development of a corresponding theoretical model deserves further investigation.

Previous studies have defined the critical mutation rate to be the midpoint between the highest mutation rate at which there is survival-of-the-fittest, and the lowest mutation rate at which there is survival-of-the-flattest (Wilke et al., 2001; Comas et al., 2005). However, the results of this study clearly show that there is a transition from survival-of-thefittest to survival-of-the-flattest and subsequently to the error catastrophe (figure 3 ).

\section{Conclusion}

This study investigated whether or not there is a relationship between population size and the size of mutation rate that can be tolerated before fitter individuals are outcompeted by those that have a greater mutational robustness (the critical mutation rate). The results show that the sizes of mutation rate at which the high, narrow peak and the lower, flatter peak are lost for increasing population sizes can be approximated by an exponential function. The effect of population size on the size of mutation rate that can be tolerated before the population loses the fittest and the flattest peaks is particularly noticeable in small populations with 100 individuals or less. This provides new insight into the factors that can affect survival-of-the-flattest in small populations, and has implications for populations under threat of local extinction. Other factors, such as sequence length and distance between peaks, may well have a significant influence on both critical mutation rate and population sizes that can withstand specific rates of mutation. It will be beneficial to investigate this in the future, as well as to construct a theoretical model (whether based on differential equations or not) that can replicate the exponential relationship between critical mutation rate and population size, found here by experiment, for low population sizes.

In addition, there is clear evidence for a continuum of mutation rates representing a transition from survival-of-thefittest to survival-of-the-flattest. This identifies a critical mutation rate by which the population has a $95 \%$ likelihood of losing the higher peak. 


\section{Acknowledgments}

This work was supported by EPSRC grant EP/H031936/1.

\section{References}

Bloom, J. D., Lu, Z., Chen, D., Raval, A., Venturelli, O. S., and Arnold, F. H. (2007). Evolution favours protein mutational robustness in sufficiently large populations. BMC Biology, 5:29.

Bornberg-Bauer, E. and Kramer, L. (2010). Robustness versus evolvability: a paradigm revisited. HFSP Journal, 4(3-4):105-108.

Bull, J. J., Meyers, L. A., and Lachmann, M. (2005). Quasispecies made simple. PLoS Computational Biology, 1(6):e61.

Comas, I., Moya, A., and González-Candelas, F. (2005). Validating viral quasispecies with digital organisms: a re-examination of the critical mutation rate. BMC Evolutionary Biology, 5:5.

Domingo, E. and Wain-Hobson, S. (2009). The 30th anniversary of quasispecies. EMBO Reports, 10:444-448.

Eigen, M., McCaskill, J., and Schuster, P. (1988). Molecular quasispecies. Journal of Physical Chemistry, 92:68816891.

Eigen, M. and Schuster, P. (1979). The hypercycle. Springer, New York.

Hartl, D. L. and Clark, A. G. (2007). Principles of population genetics. Sinauer Associates, Inc., Sunderland, MA, 4th edition.

Jagers, P. (1975). Branching processes with biological applications. Wiley.

Jones, J. and Soule, T. (2006). Comparing genetic robustness in generational vs. steady state evolutionary algorithms. In Proceedings of the 8th annual conference on genetic and evolutionary computation, pages 143-149.

Kimura, M. (1983). The neutral theory of molecular evolution. Cambridge University Press.

Kimura, M. and Maruyama, T. (1966). The mutational load with epistatic gene interactions in fitness. Genetics, 54:1337-1351.

Krakauer, D. C. and Plotkin, J. B. (2002). Redundancy, antiredundancy, and the robustness of genomes. Proceedings of the National Academy of Sciences of the United States of America, 99(3):1405-1409.

Lenski, R., Barrick, J. E., and Ofria, C. (2006). Balancing robustness and evolvability. PLoS Biology, 4:21902192.
Masel, J. and Trotter, M. V. (2010). Robustness and evolvability. Trends in Genetics, 26:406-414.

Moran, P. A. P. (1962). The Statistical Processes of Evolutionary Theory. Clarendon Press, Oxford.

Nowak, M. A. (1992). What is a quasispecies? Trends in Ecology and Evolution, 7:118-121.

Nowak, M. A. (2006). Evolutionary dynamics: Exploring the equations of life. Harvard University Press.

Nowak, M. A. and Schuster, P. (1989). Error thresholds of replication in finite populations: Mutation frequencies and the onset of Muller's ratchet. Journal of Theoretical Biology, 137:375-395.

Sanjuán, R., Cuevas, J. M., Furió, V., Holmes, E. C., and Moya, A. (2007). Selection for robustness in mutagenized RNA viruses. PLoS Genetics, 3(6):e93.

Sardanyés, J., Elena, S. F., and Solé, R. V. (2008). Simple quasispecies models for the survival-of-the-flattest effect: The role of space. Journal of Theoretical Biology, 250:560-568.

Schuster, P. (2009). Genotypes and phenotypes in the evolution of molecules. European Review, 17(2):281-319.

Takeuchi, N. and Hogeweg, P. (2007). Error-threshold exists in fitness landscapes with lethal mutants. BMC Evolutionary Biology, 7:15.

Tannenbaum, E. and Shakhnovich, E. I. (2004). Solution of the quasispecies model for an arbitrary gene network. Physical Review E, 70:021903.

Tejero, H., Marin, A., and Montero, F. (2011). The relationship between error catastrophe, survival of the flattest, and natural selection. BMC Evolutionary Biology, 11:2.

van Nimwegan, E., Crutchfield, J. P., and Huynen, M. (1999). Neutral evolution of mutational robustness. Proceedings of the National Academy of Sciences of the United States of America, 96:9716-9720.

Wilke, C. O. (2001a). Adaptive evolution on neutral networks. Bulletin of Mathematical Biology, 63:715-730.

Wilke, C. O. (2001b). Selection for fitness vs. selection for robustness in RNA secondary structure folding. Evolution, 55:2412-2420.

Wilke, C. O. (2005). Quasispecies theory in the context of population genetics. BMC Evolutionary Biology, 5:44.

Wilke, C. O. and Adami, C. (2003). Evolution of mutational robustness. Mutation Research, 522:3-11. 
Wilke, C. O., Wang, J. L., Ofria, C., Lenski, R. E., and Adami, C. (2001). Evolution of digital organisms at high mutation rates leads to survival of the flattest. $\mathrm{Na}$ ture, 412:331-333.

Wright, S. (1932). The roles of mutation, inbreeding, crossbreeding, and selection in evolution. In Proceedings of the Sixth International Congress on Genetics, pages $355-366$. 